number of outpatient clinic appointments being avoided at a cost saving to the CCG.

Conflict of Interest None

\section{EFFICACY OF DC CARDIOVERSION FOR ATRIAL FIBRILLATION: A LARGE RETROSPECTIVE OBSERVATIONAL STUDY}

Alexander Carpenter*, John Graby, Rachael Medland, Stewart Brown, Claire Sowerby, Louise Priestman, Mark Dayer, Guy Furniss. Taunton and Somerset NHS Foundation Trust

\subsection{6/heartjnl-2019-BCS.36}

Introduction The evidence base for rhythm control strategies in atrial fibrillation (AF) remain contentious. Synchronised DC cardioversion (DCCV) is a simple and accessible treatment option in many centres. However, there is little data to support its use as a long-term strategy or data regarding which groups benefit most from this strategy.

Methods We collected AF cardioversion cases across a six-year period at a busy district general hospital with a nurse-led cardioversion service. Demographic, echocardiographic and procedural data were collected, as were safety and AF outcomes at 6 and 12 months. Patients with incomplete data were excluded from the analysis. Statistical analysis was undertaken using the Fischer's exact T-test.

Results 550 cardioversion cases were included in the analysis with a median age of 67 (range 28-95). 163 (30\%) of patients were obese (BMI 30). The mean CHA2DS2-VASc was 2.3. $342(62 \%)$ of DCCV were for persistent AF, the remainder $(208 ; 38 \%)$ longstanding (AF duration $>1$ year) persistent. $162(29 \%)$ had an unknown duration of AF due to an incidental diagnosis. $483(88 \%)$ were on AV-nodal blocking and $103(19 \%)$ on anti-arrhythmic medication (flecainide, sotalol or amiodarone). 516 (94\%) of DCCVs were acutely successful with a complication rate of $2 \%(n=13)$, most commonly symptomatic bradycardia requiring temporary transcutaneous $(n=2)$ or percussion pacing $(n=6)$ with no acute strokes. At 1 year $5(0.9 \%)$ had had a stroke/transient ischaemic attack and $9(1.6 \%)$ died. 179/478 (37\%) of patients were free from AF at 6 months, with $100 / 412$ $(24 \%)$ free from $\mathrm{AF}$ at 1 year. $89(16 \%)$ of patients were referred for further cardioversion and 144 (26\%) referred for catheter ablation. There was no statistically significant effect of patient age, obesity, left ventricular (LV) impairment, or left atrial (LA) dilatation on 6-month outcomes. However, AF duration $<1$ year did correlate with statistically significant improvement in 6 month freedom from AF $(41 \%$ vs $31 \%, p=0.03)$. In those with moderate/severe LV impairment, anti-arrhythmic use significantly improved 6month outcomes $(70 \%$ vs $40 \%, \mathrm{p}=0.01)$. However, no such effect was seen between moderate/severely dilated and nondilated LA.

Conclusion DCCV for AF remains a safe procedure with good acute success rates. However, within 6 months the majority of patients will have reverted to AF. AF duration of less than 1 year is associated with an improved 6-month success rate, as does antiarrhythmic use in those with impaired LV function. In selected patients, DCCV remains a useful tool as a 'trial of sinus rhythm' to establish potential symptomatic benefits from pursuing a rhythm control strategy.

Conflict of Interest Nil

\section{INVESTIGATION OF INTERMITTENT PALPITATIONS WITH THE MICOR ECG WRISTBAND}

Birkhoelzer Sarah*, N Mullan, J Crombie, P Archer, J Gillings, Mark A Tanner. St Richard's Hospital

\subsection{6/heartinl-2019-BCS.37}

Background Palpitations are a leading cause of referral to cardiology services. The diagnosis of arrhythmia is achieved through establishing a symptom-rhythm correlation. Conventional ambulatory ECG monitoring can fail to capture symptomatic events.

Objective The ECG wristband is a wearable patient-activated lead I ECG recorder (30 seconds per event) permitting longterm monitoring and might offer a useful strategy for the initial investigation of palpitations.

Methods Patients referred to our service for investigation of paroxysmal arrhythmia were considered suitable for use of the MiCor device if they reported palpitations (or arrhythmic symptoms e.g. dizzy spells). Patients with symptoms of syncope or inability to use the device were excluded. Patients were

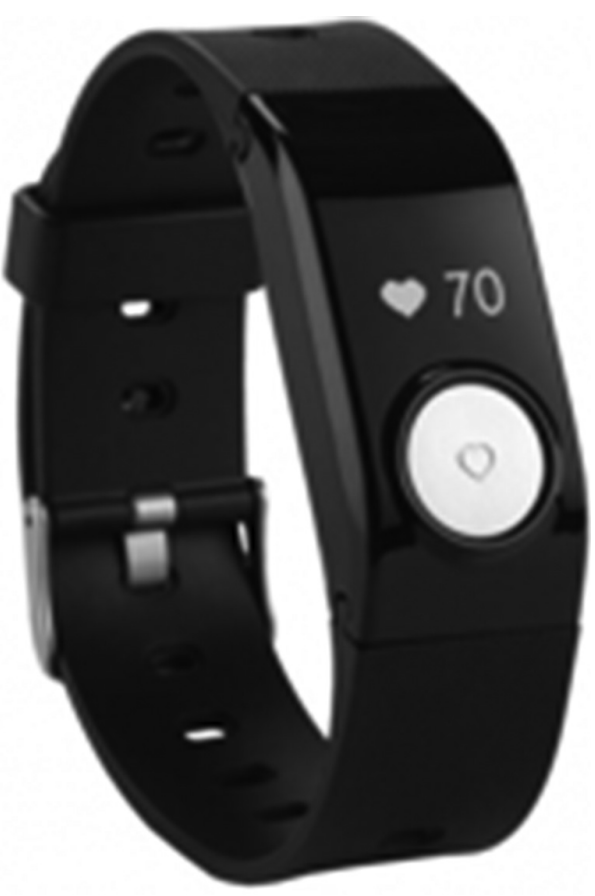

Abstract 39 Figure 1 Micor A100 wristband

Abstract 39 Table 1 Symptom-rhythm correlation $37 / 38$ patients with symptom-rhythm correlation

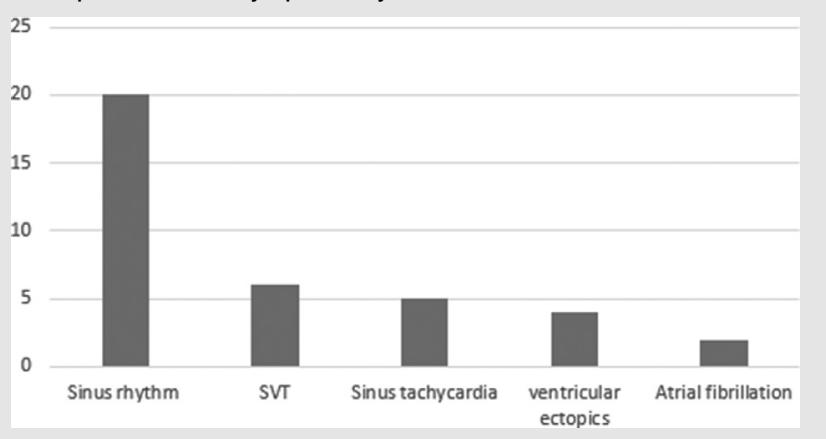


advised to activate the ECG recorder at the time of their symptoms. The ECG recordings were subsequently uploaded to a database and reviewed by a cardiologist. (Figure 1)

Results 39 patients (male 14, female 25, ages 13-86 years) were included. 38 patients submitted symptomatic recordings. A symptom-rhythm correlation was achieved in $37 / 38$ (in 1 case no ECG or symptom recording on return of device). Median time to diagnosis was 19 days (1-62). Diagnoses were: sinus rhythm 20 (54\%), sinus tachycardia 5 (13\%), ventricular ectopics 4 (11\%), SVT 6 (16\%), atrial fibrillation 2 (5\%) with time to diagnosis $16-40$ days.

Conclusion The MiCor device achieved a symptom-rhythm correlation in $97 \%$. In the majority of cases this permitted early reassurance and avoided further investigations. Where significant arrhythmia was diagnosed early implementation of therapy (including anticoagulation) was facilitated. The relative ease of use of this ECG recorder make it suitable for use in primary care where it may help reduce delays in diagnosis, improve patient experience, and reduce the number of secondary care referrals (table 1 ).

Conflict of Interest None

\section{PRE-PROCEDURE ANXIETY: THE ORIGIN OF PAIN DURING IMPLANTATION OF CARDIAC IMPLANTABLE ELECTRONIC DEVICES}

${ }^{1}$ Jonathan Senior*, 'Boyang Liu, ${ }^{2}$ Ayman Jani, 'Michail Apostolakis, ${ }^{3}$ Deepak Goyal, ${ }^{3}$ Robin Taylor, ${ }^{3}$ Will Foster, ${ }^{1}$ Nicola Brewster, ${ }^{3}$ David G Wilson. 'Worcester Royal Hospital; ${ }^{2}$ Birmingham Medical School; ${ }^{3}$ Worcestershire Acute Hospitals NHS Trust

\subsection{6/heartjnl-2019-BCS.38}

Introduction Cardiac implantable electronic device (CIED) implantation is an increasingly prevalent part of cardiac rhythm management. Adequate procedural pain management is central to the patient experience. The Association of Anaesthetists of Great Britain guidelines state that operators should develop techniques that permit the patient to undergo the procedure with minimum stress and maximum comfort. We developed an audit tool to assess the quality of pain management, and have evaluated the causative factors that can be targeted to optimise the patient experience.
Methods A prospective audit was collected over a 23-month period using a patient self-reporting questionnaire. Patients were asked to score their anxiety level prior to, and their pain during the procedure on a $0-10$ visual scale. Finally, patients were asked whether the pain matched their expectations.

Results 349 near consecutive patients $(67 \%$ male, age $75 \pm 13$ years) were included in this audit. There were 167 pacemaker implantations, 42 implantable cardioverter defibrillators, 98 cardiac resynchronisation devices, 13 lead revisions and 29 generator changes. A majority of patients $(219,63 \%)$ reported no/mild pain, with 172 (49\%) reporting the pain to be less than expected. However, 71 (21\%) patients reported the pain to be more than expected, with $56(16 \%)$ reporting severe pain.

We wanted to clarify which factors were important in influencing patients' experience of pain during their procedure. Using a logistic regression model, we investigated whether gender, weight, pre-implant anxiety, device complexity and operator predicted patients' experience of pain. Complex devices are defined as CRT, ICD, or any procedure involving lead extraction.

The logistic regression model was statistically significant $(\square 2$ 18.8, $\mathrm{P}=0.002$ ), explaining $14.8 \%$ (Nagelkerke $\mathrm{R} 2$ ) of the variance in severe pain and correctly classified $85.8 \%$ of cases. Patients with high pre-procedural worry were 2.7 times more likely to experience severe pain. Additionally, female patients were 3.2 times more likely to experience severe pain. Device complexity, operator and patient weight had no impact on pain (figure 1).

Interestingly, there was a weak positive correlation between the quantity of lidocaine usage and perceived pain $(\mathrm{R}=0.20$, $\mathrm{P}<0.001)$. It is possible that operators are using more local anaesthetic if patients are complaining of increased intra-procedural pain.

Conclusion The majority of patients underwent CIED implantation with minimum discomfort, though an important minority reported severe pain during the procedure. The most significant predictor of pain was a high level of pre-procedure anxiety and therefore interventions targeted at reducing preand peri-implant anxiety, particularly in women, warrant further investigation as a means to reduce pain during CIED implantation.

\begin{tabular}{llllllll}
\hline-5 & -3 & -1 & 1 & 3 & 5 & 7
\end{tabular}

Abstract 40 Figure 1 Forest plot demonstrating odds ratio $(95 \% \mathrm{Cl})$ derived from logistic regression for the prediction of severe pain 